\title{
Editorial
}

\section{Diet and Health}

\author{
DOI: https://doi.org/10.3329/kyamcj.v10i4.45713.
}

Diet means the kinds of food that we habitually eat or drink or take in other way for consumption in our body to live, thrive and survive. Diet has utmost intimate relationship with our overall health and healthy life. Without a balanced diet, none can have a good health and healthy life. With inadequate or inappropriate excessive diet, one gets ill with substantial increase in morbidity and mortality.

Nutrition means the portion of food required for sustenance of health and life, growth, reproduction and to combat diseases. Poor nutritions mean (1) under-eating or (2) over-eating or (3) malnutrition (not getting optimum healthy food items we need each day, or taking too many types of food and drink, which are low in fibre or high in fat, salt and/or sugar). Poor nutrition badly impairs our abilities to combat stresses, our capacity to work causing tiredness or incapacitating us, and over time, it leads to several illnesses and other health problems such as: being overweight or obese, dental caries, hypertension hyperlipemia, coronary and cerebrovascular diseases, diabetes mellitus (DM), osteoporosis, malignancies (specially of breast, colon and pancreas etc.), gallbladder diseases, osteoarthritis, gout, sleep apnea, respiratory diseases, psychiatric disorders like depression etc, along with their attendant complications. ${ }^{1}$

Surprisingly when many people of underdeveloped countries like those from Africa, Asia and migrating refugees become ill and even die of undernutrition and malnutrition, even more people from the rest of the world become ill and die from socalled aristocratic disease of obesity mostly because of overeating. The latter group of people consume diet rich in saturated fats, partially-hydrogenated oils, refined carbohydrates, and highly processed foods. This is more true for people with sedentary life styles. People whose dietary patterns include fresh, whole foods like fruits and vegetables, whole grains, legumes, lean meats, and fish have a lower incidence of major chronic disease and especially of dietrelated diseases. $^{2}$

Eating fats isn't always bad. Our body needs some fats to remain healthy. We need the correct kind of fat in adequate and optimum amount to remain healthy and to prevent diseases. We should get our fats from vegetables and fishes, minimizing saturated fats from other animal sources. Saturated and trans fats typically have been understood as 'bad', while monounsaturated fats and polyunsaturated fats are generally classified as 'good'. ${ }^{3}$ Fast food is appealing since it's cheap, convenient, tasty, and in many circles, socially acceptable. But the health concerns are dramatic. Fast food and processed foods are being designed to alter our brains and drive food addiction. The science of food addiction says these foods produce the same biochemical effects in the brain that are characteristic of substance abuse. ${ }^{4}$

Lots of junk food, fast food, processed foods also contribute to becoming overweight and obese. In obesity, adverse effects on health occurs owing to excess accumulated body fat.

Obesity is now the number one cause of preventable death in many developed countries. Obesity causes loss of more than 47 per cent life years than tobacco abuse. Dr. Tiwet named twenty seven medical issues that a poor diet/obesity is known to cause or is highly suspected to contribute to: 1 . Hypertension, 2. Alzheimer's disease, 3. Coronary Heart Disease, 4. CerebroVascular Disease, 5. Gall Bladder Disease , 6. Osteoarthritis, 7. Sleep Apnea, 8. Respiratory Problems, 9. Endometrial Cancer, 10. Breast Cancer, 11. Prostate Cancer, 12. Colon Cancer, 13. Dyslipidemia, 14. Nonalcoholic Steatohepatitis, 15. Insulin Resistance, 16. Asthma 17. Hyperuricemia, 18. Reproductive Hormone Abnormalities, 19. Polycystic Ovarian Syndrome, 20. Impaired Fertility, 21. Type-2 (Adult Onset) DM, 22. Depression, 23. Anxiety, 24. Low Energy Levels/Fatigue, 25. Tooth Decay, 26. Acne, 27. Digestive Health Issues.

In addition, excess diet may increase the risks of pregnancy problems in the form of hyperglycemia, hypertension and increased risk for caesarean delivery, etc. ${ }^{4}$

Optimum amount of carbohydrate intake is a must, especially to avoid too much sweet stuffs containing sugar. Consuming a large amount of processed sugar can trigger feelings of worry, irritability, and sadness that can aggravate depression, anxiety disorders and panic attacks. Fructose from sugary items such as soda slows down brain, which can hamper memory and learning. Genes in the brain could be damaged by fructose. All these can lead to or impact Alzheimer's disease, diabetes, and heart disease. ${ }^{6}$

'Eating Disorders' are psychiatric disorders of abnormal eating that negatively impairs physical and mental health. They include 'Binge Eating Disorder', 'Anorexia Nervosa', 'Bulimia Nervosa', 'Pica', 'Rumination Disorder' 'Avoidant/Restrictive Food Intake Disorder' and many other specified eating or feeding disorders. These disorders don't include obesity. Effective treatment may be possible by counseling, proper diet, exercise etc. with or without hospitalization and medication. Prognosis is variable with increased risks of death in case of some disorders.

Children who consume nutrient-rich diets have been found to perform better academically than those who are nutrientdeficient. Well-fed children have been found to be lesshyperactive, less moody, more cooperative. Children are also developing habits and tastes that will affect them as they become adults. If kids eat fast foods often, it will be harder for them to break those patterns later in life. And childhood eating habits have far-reaching consequences. Eating candy and sweets in childhood is linked to later-life violence, drug use, and drug abuse more than any other parameter. when children eat unhealthy foods, they may be damaging their genes. And raising children on unhealthy foods doesn't only impact their lives, but also the lives of their descendants. ${ }^{8}$ 


\section{References}

1. Gray DS, Fujioka K. Use of relative weight and Body Mass Index for the determination of adiposity. Journal of Clinical Epidemiology. 1991; 44 (6): 545-550.

2. Katherine Zeratsky, R.D, L.D. Normal weight obesity: A hidden health risk?: Can you be considered obese if you have a normal body weight? Mayo Clinic-Obesity Expert Answers. [ Internet] 2009 Aug. 5. [Retrieved 2010 Jan 25]

3. Romero-Corral, A. Somers, V. Lopez-Jimenez, F. Korenfeld, Y. Palin, S. Boelaert, et al. 3-D Body Scanner, Body Volume Index: A Novel, Reproducible and Automated Anthropometric Tool Associated with Cardiometabolic Biomarkers Obesity A Research Journal 2008; 16 (1): 266.

4. World Health Organization. BMI classification, [Retrieved 2014 Feb15]
5. Jebb S, Wells J. Measuring body composition in adults and children. In:Peter G. Kopelman, Ian D Caterson, Michael J Stock, William H. Dietz. Clinical obesity in adults and children: In Adults and Children. Blackwell Publishing; 2005. 12-28.

6. Kopelman P, Caterson I, An overview of obesity management In:Peter G. Kopelman, Ian D. Caterson; Michael J Stock, William H. Dietz Clinical obesity in adults and children: In Adults and Children. Blackwell Publishing; 2005.319-326.

7. Seidell JC. Epidemiology- definition and classification of obesity. In:Peter G. Kopelman; Ian D. Caterson; Michael J Stock, William H. Dietz Clinical obesity in adults and children: In Adults and Children. Blackwell Publishing; 2005. 3-11.

8. Puhl R, Henderson K, and Brownell K. Social consequences of obesity In:Peter G. Kopelman; Ian D. Caterson, Michael J, Stock, William H. Dietz Clinical obesity in adults and children: In Adults and Children. Blackwell Publishing; 2005.29-45.

\section{ABM Moniruddin}

Professor

Department of Surgery 